\title{
Convective rolls and heat transfer in finite-length Rayleigh-Bénard convection: A two-dimensional numerical study
}

\author{
S. Kenjereš and K. Hanjalić \\ Department of Applied Physics, Thermo-Fluids Section, Delft University of Technology, Lorentzweg 1, 2628 CJ Delft, The Netherlands
}

(Received 12 June 2000)

\begin{abstract}
A two-dimensional (2D) numerical study using a single-point algebraic $k-\bar{\theta}^{2}-\varepsilon-\varepsilon_{\theta}$ turbulence closure was performed to detect the existence, origin, creation and behavior of convective rolls and associated wall Nusselt $(\mathrm{Nu})$ number variation in thermal convection in 2D horizontal slender enclosures heated from below. The study covered the Rayleigh (Ra) numbers from $10^{5}$ to $10^{12}$ and aspect ratios from 4:1 to 32:1. The time evolution of the convective rolls and the formation of the corner vortices were analyzed using numerical flow visualization, and the correlation between roll structures and heat transfer established. A major consequence of the imposed two dimensionality appeared in the persistence of regular roll structures at higher Ra numbers that approach a steady state for all configurations considered. This finding contradicts the full three-dimensional direct numerical simulations (DNS), large eddy simulations (LES), and three-dimensional transient Reynolds-averaged Navier-Stokes (TRANS) computations, which all show continuously changing unsteady patterns. However, the final-stage roll structures, long-term averaged mean temperature and turbulence moments, and the Nusselt number (both local and integral), are all reproduced in good agreement with the ensemble-averaged 3D DNS, TRANS, and several recent experimental results. These findings justified the 2D approach as an acceptable method for ensemble average analysis of fully 3D flows with at least one homogeneous direction. Based on our 2D computations and adopting the low and high Ra number asymptotic power laws of Grossmann and Lohse [J. Fluid Mech. 407, 27 (2000)], new prefactors in the Nu-Ra correlation for $\operatorname{Pr}=\mathcal{O}(1)$ were proposed that fit better several sets of data over a wide range of $\mathrm{Ra}$ numbers and aspect ratios: $\mathrm{Nu}=0.1 \mathrm{Ra}^{1 / 4}+0.05 \mathrm{Ra}^{1 / 3}$. Even better agreement of our computations was achieved with the new correlation $\mathrm{Nu}=0.124 \mathrm{Ra}^{0.309}$ proposed recently by Niemela et al. [Nature (London) 404, 837 (2000)] for $10^{6} \leqslant \mathrm{Ra} \leqslant 10^{17}$.
\end{abstract}

PACS number(s): 47.27.- i, 05.60.-k

\section{INTRODUCTION}

Buoyancy driven flows heated from below are characterized by large cellular structures (convection roll cells), which act as a major heat and momentum carrier and communicator between the bottom heated and top cooled wall. The presence of roll cell structure is confirmed in laminar and lowturbulence intensity regimes, both experimentally and by direct numerical simulations (DNS). On the other hand, very little is known about these structures at high Rayleigh (Ra) numbers. With an increase in Ra, the flow becomes more turbulent in the complete domain and the arrays of roll cells become highly unsteady. Thermals released from the boundary layers at the bottom hot and upper cold horizontal surfaces, penetrating upwards and downwards towards the opposite wall of lower or higher temperature, cause very intensive mixing in the central region, creating in long-term average an almost isothermal core. Until very recently, the existence of organised convection roll cells had not been detected by experiments at high Ra number. One of the first indications about their existence in turbulent RayleighBénard convection can be found in the experimental work of Chu and Goldstein [1]. In their experiment at low-Ra number with water they observed that many thermals retain their original identity even after arriving at the near wall region of the opposite surface. These thermals coalesce and form stable patterns with a temperature different from the temperature of the surrounding fluid. These stable patterns provided an explanation for the phenomenon of inversion of the temperature profile, recorded earlier experimentally. From the interferograms, Chu and Goldstein [1] also concluded that the thermals were periodically released from relatively fixed locations. In addition, they noticed that these active periods at fixed sites become more regular as Ra increases and that "a persistent horizontal motion near the horizontal boundaries" exist. Although they did not so state explicitly, it seems obvious that the neighboring thermals, moving in opposite directions entrain the surrounding fluid and generate a circular motion in the space between. This movement leads in fact to the creation of a pattern of roll cells. These structures, although highly unsteady, can give an ensemble averaged structure pattern very similar to that found in the laminar regime. In contrast to the long-term mean properties averaged over the homogeneous horizontal planes, which at high Ra numbers vary only in the vertical direction, we postulate that such roll cell patterns exist as long-term ensemble averages, with a corresponding strong variation of the wall heat transfer and flow properties in the horizontal direction. This pattern is particularly noticeable in enclosures of smaller aspect ratios when the side walls tends to enhance the creation of roll cell like motion.

Experimental evidence was recently significantly updated in the range of moderate and high Ra numbers. In a series of experiments by the Chicago group, Heslot et al. [2], Castaing et al. [3], where an enclosure with an aspect ratio of one was considered, the different states of turbulence were observed for low and high Ra numbers. However, unlike the previous theories, which led to the classical $\mathrm{Nu} \propto \mathrm{Ra}^{1 / 3}$ relationship, 
the new results for $\mathrm{Ra}>10^{5}$ seem to follow closer the $2 / 7$ power law over a wider range of Ra numbers, at least in so called hard regime for $\mathrm{Ra}>4 \times 10^{7}$. An explanation was offered in the evidence of the existence of the horizontal boundary layers at much higher Ra numbers than hitherto acknowledged. Chavanne et al. [4-6] repeated some of the experiments performed by the Chicago group and increased $\mathrm{Ra}$ up to $5 \times 10^{12}$. In addition to the previously mentioned different states of turbulence, they observed a new "ultimate" regime in which the exponent of the Ra number increases at least to 0.4 .

Despite simplification in geometry and boundary conditions (constant temperature of horizontal thermally active walls) experimental studies are always faced with difficulties in detecting the flow and turbulence details, particularly in the near wall regions, and with uncontrolled heat losses. In contrast to experiments, numerical studies can provide detailed insight in flow and heat transfer behavior at desired boundary conditions. However, direct numerical simulations (DNS) are faced with different limitations. For example, Kerr [7] addresses many numerical problems in DNS, even for the classical case of Rayleigh-Bénard convection (infinite enclosures). He demonstrated that numerical solvers were not sufficiently efficient to represent the rigid walls in more than one direction. The numerical simulation based on the vorticity-stream function approach was used in the work of Ishiwatari et al. [8]. They investigated effects of various combinations of thermal boundary conditions on the pattern of two-dimensional convection. The adopted thermal boundary conditions included different combinations of fixed temperature and/or fixed heat flux with or without internal cooling. The first target of their investigation was the pattern and horizontal scales of the convective rolls. They compared their results with earlier ones from the literature and found controversial results regarding the size and ratio between the vertical and horizontal scales of convective rolls. Rothermel and Agee [9] found at $\mathrm{Ra}=600 \mathrm{Ra}_{c}$ convective rolls with a horizontal scale about ten times larger than the layer depth in their two-dimensional calculations. Contrary to their work, Sykes and Henn [10] did not detect such large horizontal scales. The results of Ishiwatari et al. [8] confirmed that convective rolls with larger horizontal size tend to form in situations where the heat flux was applied as the thermal boundary condition at the wall. Chapman et al. [11] and Chapman and Proctor [12] reported similar findings, concluding that with an increase in Ra number, the roll cells became more elongated. They compared their numerical results with the weak nonlinear description of the formation of horizontally elongated rolls and found that this approach is applicable only when $\mathrm{Ra} \approx \mathrm{Ra}_{c}$. The results of Hewitt et al. [13] showed that the horizontal scale of convective rolls grows gradually as the $\mathrm{Ra}$ increases. A similar conclusion was reached by Trompert and Hansen [14].

The above brief overview of selected literature indicates a number of interesting but controversial findings concerning flow pattern and size of convective rolls. It is important to note that all numerical studies mentioned were performed in the range of low and moderate Ra numbers. Our goal was to extend this range to high Ra numbers and to analyze effects of the aspect ratio and the side walls on roll/cell structures. Another goal was to check the applicability of a two- dimensional approach to provide ensemble-averaged solutions of an essentially three-dimensional problem: if one can capture local variation of $\mathrm{Nu}$ numbers together with a longterm average quantities with a two-dimensional simulation, the $2 \mathrm{D}$ approach will be a great advantage in terms of computational costs [saving of at least $\mathcal{O}\left(10^{2}\right)$, since this is a usual number of grid points in the horizontal direction]. Of course, the 2D approach cannot resolve the turbulence spectral dynamics even if the Navier-Stokes equations are fully resolved in time, since one of the essential process, that of vortex stretching, is missing. This is compensated by the turbulence model, by which the ensemble-averaged 2D Navier-Stokes equations are closed. The 2D approach may be even more questionable in direct numerical simulations. Yet, several publications on 2D simulation of thermal convection in slender and square enclosures, e.g., Goldhirsh et al. [15], Ishieatari et al. [8], Paolucci [16] mimicked well the experimental observations of the mechanism of roll formation and provided a physical insight into their dynamics that is of relevance also to real $3 \mathrm{D}$ situations.

The paper presents first the applied turbulence model and numerical method: we demonstrate the inadequacy of isotropic eddy diffusivity approach and illustrate the importance of applying at least a simplified algebraic model to capture the basic physics in an enclosure with 1:8 aspect ratio at $\mathrm{Ra}$ $=10^{7}$. Next we present a compilation of a number of experimental DNS, LES, and TRANS results for mean temperature and Nusselt numbers from the literature and compare our computations. Local Nusselt number variation along the walls is discussed for various aspect ratios. A new Nu-Ra number correlation for long-term averaged heat transfer is proposed that seem to fit well data from various sources and our computations over a wide range of Rayleigh numbers. Finally, we discuss the time evolution and formation of rolls, and the ultimate rolls pattern.

\section{MODEL AND NUMERICAL METHOD}

The mathematical model of turbulence and the numerical method used have been published earlier and here we give only a brief summary. More details can be found in Hanjalić [17], Kenjereš and Hanjalić [18], Hanjalić, Kenjerešs, and Durst [19], and Kenjereš [20].

The Reynolds-averaged momentum and energy equations governing the turbulent flow subjected to thermal buoyancy are

$$
\begin{gathered}
\frac{D U_{i}}{D t}=F_{i}-\frac{1}{\rho} \frac{\partial P}{\partial x_{i}}+\frac{\partial}{\partial x_{j}}\left(\nu \frac{\partial U_{i}}{\partial x_{j}}-\overline{u_{i} u_{j}}\right), \\
\frac{D T}{D t}=\frac{q}{\rho c_{p}}+\frac{\partial}{\partial x_{j}}\left(\frac{\nu}{\sigma_{T}} \frac{\partial T}{\partial x_{j}}-\overline{\theta u_{j}}\right),
\end{gathered}
$$

where $D / D t=\partial / \partial t+U_{k} \partial / \partial x_{k}$ is the material derivative, $F_{i}$ is the body force acting on the fluid (here thermal buoyancy), and $q$ is the internal energy sources.

The turbulent flux of heat $\overline{\theta u_{j}}$ is provided by the algebraic flux model (denoted in figures as AFM) obtained by truncation of the parent model differential transport equations for $\overline{\theta u_{j}}$. The so called reduced model was used which follows from a full neglect of the transport terms for $\overline{\theta u_{j}}$, i.e., 
$(D / D t-\mathcal{D}) \overline{\theta u_{i}}=0$, where $\mathcal{D}$ stands for diffusion. This essentially means that the production and dissipation of both $k$ and $\bar{\theta}^{2}$ are locally in balance. The final expression for the turbulent heat flux is

$$
\overline{\theta u_{i}}=-C^{\theta} \frac{k}{\varepsilon}\left(\overline{u_{i} u_{j}} \frac{\partial T}{\partial x_{j}}+\xi \overline{\theta u_{j}} \frac{\partial U_{i}}{\partial x_{j}}+\eta \beta g_{i} \bar{\theta}^{2}+\varepsilon_{\theta i}\right) .
$$

Although substantially simplified, Eq. (3) still reflects the basic physics, since it retains all three production terms from the differential transport equation for $\overline{\theta u_{j}}$ representing physical mechanisms which generate the turbulent heat flux: due to the nonuniformity of the mean thermal field $\nabla T$, mechanical deformation (mean rate of strain $\nabla U_{i}$ ) and amplification/ attenuation of turbulence fluctuations due to the effect of buoyancy $\beta g_{i} \bar{\theta}^{2}$, respectively. The last term in the Eq. (3), $\varepsilon_{\theta i}$, representing the molecular dissipation of $\overline{\theta u_{i}}$ is omitted as negligible at high Ra numbers (cross correlation of derivatives of fluctuating temperature and velocity), as compared with the major sink due to pressure scrambling.

It is noted that neglecting the last two production terms leads to the nonisotropic eddy-diffusivity model [known also as the generalized gradient diffusion hypothesis (GGDH)] which can be regarded as a further step in the hierarchy of truncation of the differential transport equation for $\overline{\theta u_{i}}$. Finally, replacing the turbulent stress $\overline{u_{i} u_{j}}$ by its trace $\overline{u_{i} u_{i}}$ $=2 \mathrm{k}$ leads to the standard isotropic eddy-diffusivity model, known also as the simple gradient diffusion hypothesis (SGDH):

$$
\overline{\theta u_{i}}=-\frac{\nu_{t}}{\sigma_{T}^{t}} \frac{\partial T}{\partial x_{i}}
$$

Although SGDH is used in most CFD computations, it should be noted that Eq. (4) assumes that the vertical turbulent heat flux is proportional to the mean temperature gradient in the vertical direction, which in most part of enclosures heated from below is zero (or even slightly negative, i.e., with temperature inversion). Hence, in order to reproduce the mean temperature and the ensemble averaged roll pattern, it is of crucial importance to use an algebraic expression for $\overline{\theta u_{i}}$, which includes all production terms, as shown by Kenjereš [20]. This is not possible with any type of eddy diffusivity models, be they isotropic (SGDH) or the nonisotropic (GGDH).

For the momentum flux, we used the standard eddyviscosity model $\overline{u_{i} u_{j}}=\frac{2}{3} k \delta_{i j}-\nu_{t}\left(\partial U_{i} / \partial x_{j}+\partial U_{j} / \partial x_{i}\right)$, where $\nu_{t}$ is the eddy viscosity, defined as $C_{\mu} f_{\mu} k^{2} / \widetilde{\varepsilon}$. The use of an eddy-viscosity model for turbulent stresses and an algebraic model for the turbulent heat flux may sound inconsistent. Indeed, we switched later to the consistent approach using also the reduced algebraic stress model and repeated the computations of some cases. The difference was only marginal. An explanation can be found in the fact that the two horizontal stress components in the horizontal homogeneous planes are equal and significantly smaller than the vertical component, which contributes most to the kinetic energy and is also most responsible for the vertical heat transfer on both walls. Hence, the eddy viscosity approach with ki- netic energy serving as the velocity scale seems adequate for the slender horizontal enclosures. However, for more general cases, we certainly recommend the fully consistent algebraic approach.

The closure of the algebraic expression, irrespective of the modeling level, requires that the basic scalar variables $k$, $\bar{\theta}^{2}, \widetilde{\varepsilon}$, and $\widetilde{\varepsilon}_{\theta}$ be provided from separate modeled transport equations. Because no universal wall functions have ever be proposed for buoyancy driven flows, the integration of all equations need to be performed up to the wall and the model equations must account for molecular effects that prevail in the near-wall viscous or conductive regions. The resulting four-equation $k-\varepsilon-\bar{\theta}^{2}-\varepsilon_{\theta}$ model (for details, see, e.g., Hanjalic [17]) consists of the following equation set:

$$
\begin{gathered}
\frac{D(\rho k)}{D t}-\mathcal{D}_{k}=\rho P+\rho G-\rho \varepsilon, \\
\frac{D\left(\rho \bar{\theta}^{2}\right)}{D t}-\mathcal{D}_{\theta}=2 \rho P_{\theta}-2 \rho \varepsilon_{\theta}, \\
\frac{D(\rho \tilde{\varepsilon})}{D t}-\mathcal{D}_{\varepsilon}=\frac{\tilde{\varepsilon}}{k} \rho\left(C_{\varepsilon 1} P+C_{\varepsilon 3} G\right)-C_{\varepsilon 2} f_{\varepsilon} \rho \frac{\tilde{\varepsilon}^{2}}{k}+E+S_{l}, \\
\frac{D\left(\rho \widetilde{\varepsilon}_{\theta}\right)}{D t}-\mathcal{D}_{\varepsilon \theta}=C_{\varepsilon 1}^{\theta} \rho P{ }_{\theta} \frac{\widetilde{\varepsilon}_{\theta}}{\bar{\theta}^{2}}+C_{\varepsilon 3}^{\theta} \rho P \frac{\widetilde{\varepsilon}_{\theta}}{k}-C_{\varepsilon 4}^{\theta} \rho \frac{\widetilde{\varepsilon}_{\theta}^{2}}{\bar{\theta}^{2}} \\
-C_{\varepsilon 5}^{\theta} \rho f_{\varepsilon \theta} \frac{\tilde{\varepsilon}_{\theta} \tilde{\varepsilon}}{k}+E_{\theta},
\end{gathered}
$$

where

$$
\begin{gathered}
P=-\overline{u_{i} u_{j}} \frac{\partial U_{i}}{\partial x_{j}}, \quad G=-\beta g_{i} \overline{\theta u_{i}}, \quad P_{\theta}=-\overline{\theta u_{j}} \frac{\partial T}{\partial x_{j}}, \\
E=2 \mu \frac{\mu_{t}}{\rho}\left(\frac{\partial^{2} U_{i}}{\partial x_{j} \partial x_{k}}\right)^{2}, \quad E_{\theta}=2 \rho a a_{t}\left(\frac{\partial^{2} T}{\partial x_{j} \partial x_{k}}\right)^{2}, \\
\tilde{\varepsilon}=\varepsilon-2 \nu\left(\frac{\partial k^{1 / 2}}{\partial x_{k}}\right)^{2}, \quad \tilde{\varepsilon}_{\theta}=\varepsilon_{\theta}-\alpha\left(\frac{\partial \bar{\theta}^{2} 1 / 2}{\partial x_{k}}\right)^{2}, \\
S_{l}=\max \left[0.83 \frac{\varepsilon^{2}}{k}\left(\frac{k^{3 / 2}}{C_{l} \varepsilon x_{n}}-1\right)\left(\frac{k^{3 / 2}}{C_{l} \varepsilon x_{n}}\right)^{2}, 0\right] \\
\mathcal{D}_{\phi}=\frac{\partial}{\partial x_{j}}\left(\mu \frac{\partial \phi}{\partial x_{j}}+C_{\phi} f_{\mu} \rho \frac{k^{2}}{\tilde{\varepsilon}} \frac{\partial \phi}{\partial x_{j}}\right)^{2}, \\
f_{\mu}=\exp \left[\frac{-3.4}{\left(1+\frac{R e_{t}}{50}\right)^{2}}\right], \quad f_{\varepsilon}=1-0.3 \exp \left(-R e_{t}^{2}\right), \\
f_{\varepsilon \theta}=1
\end{gathered}
$$

and index $\phi$ in equation for $\mathcal{D}_{\phi}$, Eq. (9), stands for any of the variables for which the transport equations are solved. 
TABLE I. Specification of coefficients.

\begin{tabular}{llllllllll}
\hline \hline$C^{\theta}$ & $C_{\theta^{2}}$ & $C_{\varepsilon \theta}$ & $C_{\varepsilon 1}^{\theta}$ & $C_{\varepsilon 3}^{\theta}$ & $C_{\varepsilon 4}^{\theta}$ & $C_{\varepsilon 5}^{\theta}$ & $C_{l}$ & $\xi$ & $\eta$ \\
\hline 0.2 & 0.09 & 0.09 & 1.3 & 0.72 & 2.2 & 0.8 & 2.5 & 0.6 & 0.6 \\
\hline \hline
\end{tabular}

In addition to standard coefficients in the $k$ and $\varepsilon$ equations $\left(C_{k}=0.09, \quad C_{\varepsilon}=0.07, \quad C_{\varepsilon 1}=C_{\varepsilon 3}=1.44\right.$, and $C_{\varepsilon 2}$ $=1.92$ ), the remaining coefficients used are specified in Table I.

A further simplification can be achieved by expressing $\varepsilon_{\theta}$ in terms of $\varepsilon, k$, and $\bar{\theta}^{2}$ from the assumed ratio of the thermal to mechanical turbulence time scales $R=\tau_{\theta} / \tau_{m}$ $=\bar{\theta}^{2} \varepsilon /\left(2 k \varepsilon_{\theta}\right)$ with either $R=$ const, or prescribed by an algebraic function in terms of available variables. This reduces the model to a three-equation one, $k-\varepsilon-\bar{\theta}^{2}$ which has five coefficients less. It is worth mentioning that the three equation model $k-\varepsilon-\bar{\theta}^{2}$ with $R=0.5$ gives very similar results as the four-equation model. However, for completeness, we present here the results with the four-equation model.

The simulations were performed by a version of finite volume Navier-Stokes solver for two-dimensional flows in Cartesian coordinates. A staggered grid was used, clustered in the vicinity of the vertical and horizontal wall. The number of grid cells was between 80 and 260 in the vertical direction and between 220 and 820 in the horizontal direction. Larger values correspond to higher $\mathrm{Ra}$ number and larger aspect ratios. More details on the grid are given in descriptions of various cases considered.

The convection terms were computed by the second order linear-upwind (LUDS) scheme, and the diffusion terms by the central-difference schemes (CDS). The time integration was performed by the implicit three-level second order marching time difference scheme with the nondimensional time step of $1 / 50-1 / 100 \tau^{*}$, where $\tau^{*}=\tau \sqrt{\beta g \Delta T H} / H$ is the characteristic convective time of the rolls.

\section{RESULTS AND DISCUSSION}

\section{A. Flow pattern, mean, and turbulence variables}

As a representative of slender geometries, we consider first an enclosure of $1: 8$ aspect ratio at $\mathrm{Ra}=10^{7}$ and 2 $\times 10^{9}$. Figures 1,2 , and 3 show the computed mean velocity vectors, mean temperature, and several turbulence parameters: temperature variance $\bar{\theta}^{2}$, horizontal and vertical components of the turbulent heat flux $\overline{\theta u_{i}}$, turbulence kinetic energy $k$, and turbulence Reynolds number $\operatorname{Re}_{t}=k^{2} / \nu \tilde{\varepsilon}$. It is recalled that $\mathrm{Re}_{t}$ is proportional to the ratio of the turbulent to molecular viscosity: if $\nu_{t}=C_{\mu} k^{2} / \widetilde{\varepsilon}$, with $C \mu=0.09$ as in the standard high-Reynolds-number $k-\varepsilon$ model $\operatorname{Re}_{t}$ $\approx 10 \nu_{t} / \nu$.

The roll patterns are visible in all three figures, though with different modes. One can also identify the ensembleaveraged formations of thermals as well as horizontal motions along the thermally active lower and upper walls, resembling the interferograms of Chu and Goldstein [1], and the visualization photographs of Theerthan and Arakeri [21] and Theerthan [22]. The organised roll structure causes a strong periodic variation of the local $\mathrm{Nu}$ numbers along the
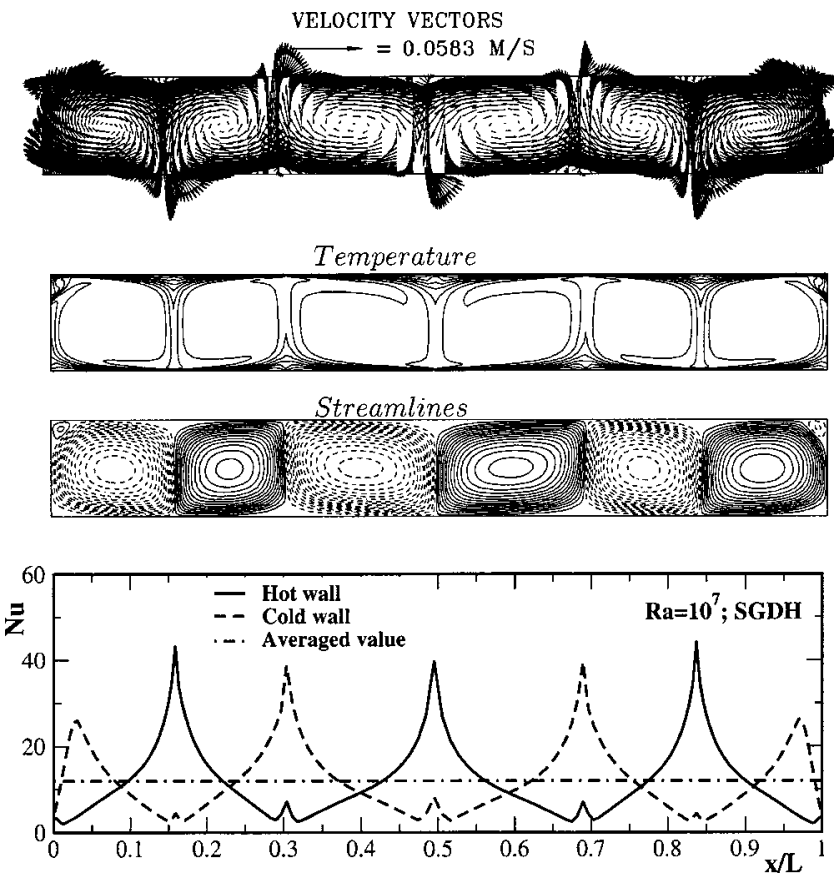

FIG. 1. SGDH solutions for 1:8 enclosure heated from bottom for $\mathrm{Ra}=10^{7}$, laminarised solution.

horizontal walls with sharp maximum values in the stagnation points where the plume impinges on the opposite wall, penetrating the wall boundary layer. In contrast, the minimum values of $\mathrm{Nu}$ number correspond to the positions where the plume release occurs, where the fluid, moving horizontally in the wall boundary layers, accumulates and where the boundary layer is thickest.

Figures 1 and 2 show the computed results for the same Rayleigh number $\mathrm{Ra}=10^{7}$, but obtained with two turbulence models SGDH and AFM. It is important to note that the SGDH results gave a laminarised solution, i.e., the isotropic eddy diffusivity employed by the SGDH model cannot maintain turbulence irrespective of the initial turbulence field. In contrast, the results for the same configuration computed by the AFM shown in Fig. 2 yielded the turbulent solution. No detailed experimental nor DNS data are available but the validation of the computations can be made by comparing the long-term averaged $\mathrm{Nu}$ numbers with experimental correlations. The $\mathrm{SGDH}$ gave $\overline{\mathrm{Nu}}=12$ which is in agreement with correlation for laminar regime, whereas AFM gave $50 \%$ higher value, $\overline{\mathrm{Nu}}=18$ in accord with the correlation for a turbulent regime. It is interesting to see that in the laminar solution obtained by SGDH, the local $\mathrm{Nu}$ number distributions show sharper peaks than in the case of the turbulent AFM solution. The amplitude of the local $\mathrm{Nu}$ numbers at impingement locations are $20 \%$ higher than in the AFM case. It means that at particular locations, the temperature gradients are higher than in the AFM case, indicating weak mixing. In the case of the AFM results, the turbulent solution provides a better mixing which decreases the temperature differences in the enclosure producing milder local peaks, but significantly higher averaged value of the $\mathrm{Nu}$ number.

With an increase in the Ra number, Fig. 3, the variation in the local Nusselt numbers becomes milder with the wavelength increasing. Also, the wall thermal boundary layers become much thinner and the maximum values of the tem- 

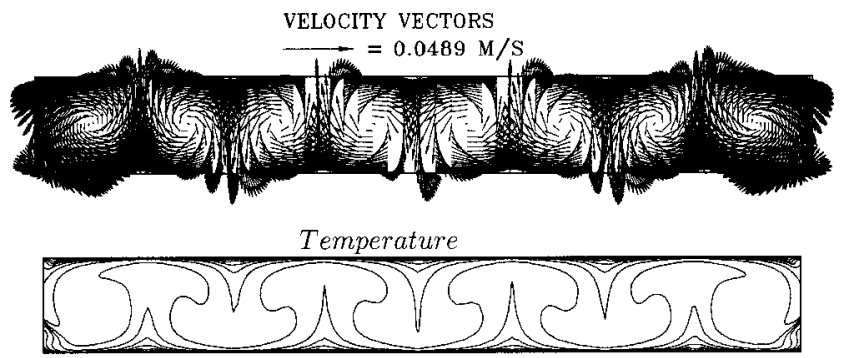

$\overline{\theta^{2}}$

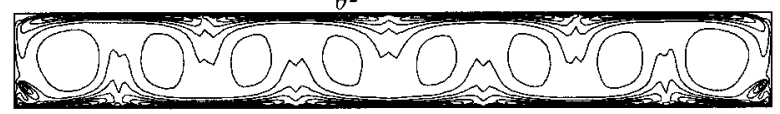

$\overline{\theta u}$

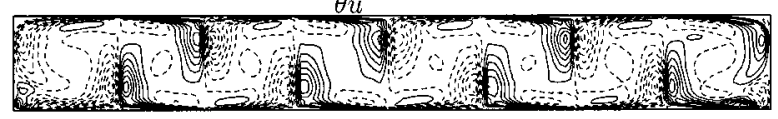

$\overline{\theta v}$
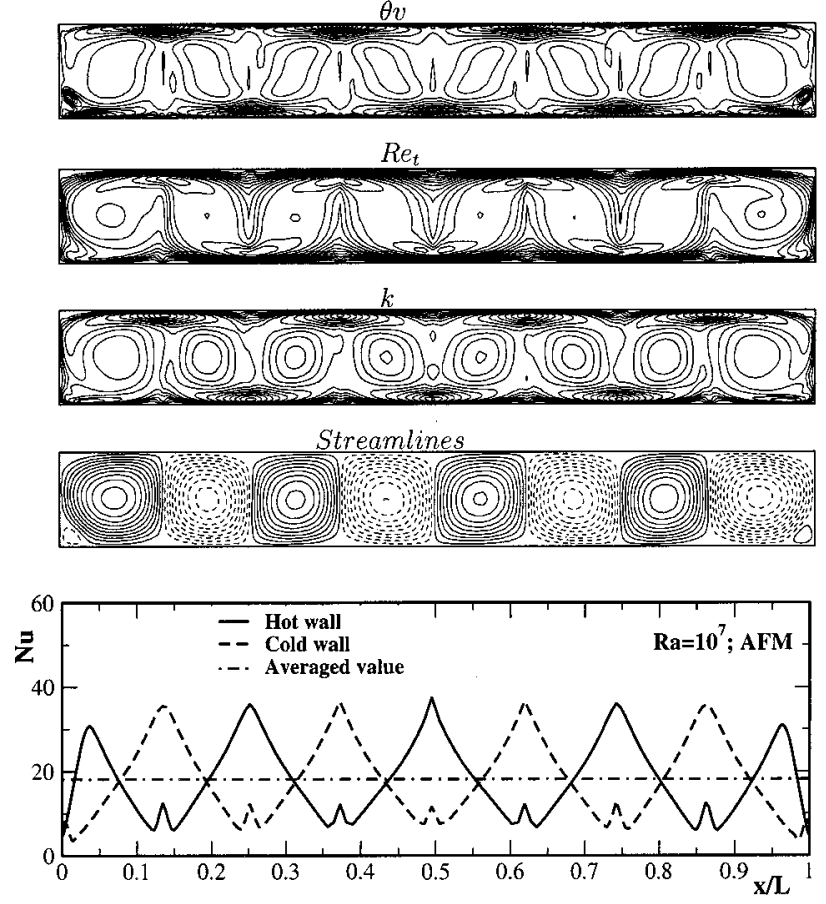

FIG. 2. AFM solutions for 1:8 enclosure heated from bottom for $\mathrm{Ra}=10^{7}$.

perature fluctuations are concentrated in relatively small near-wall regions as compared with the case at lower value of the Ra number, Fig. 2. The distributions of the turbulent Reynolds numbers show different behavior for different values of the Ra number. For the low Ra number, the maximum values are concentrated in the impingement regions, whereas in the high Ra case in the middle of the enclosure indicating higher intensity of mixing.

Figure 4 shows the ultimate ensemble averaged patterns in two-dimensional 1:4 aspect ratio enclosure for two values of the $\mathrm{Ra}$ number, $\mathrm{Ra}=10^{7}$ and $\mathrm{Ra}=2 \times 10^{9}$. For lower $\mathrm{Ra}$ number, the flow pattern consists of four large and two small corner rolls. For the higher $\mathrm{Ra}$ number, $\mathrm{Ra}=2 \times 10^{9}$, the flow pattern consists of two large and two small corner rolls. The final state is symmetric with respect to the cavity centreline for both values of the Ra number. As in the case of the higher aspect ratio 1:8 the near-wall boundary layers along the horizontal walls are visible and determined by the
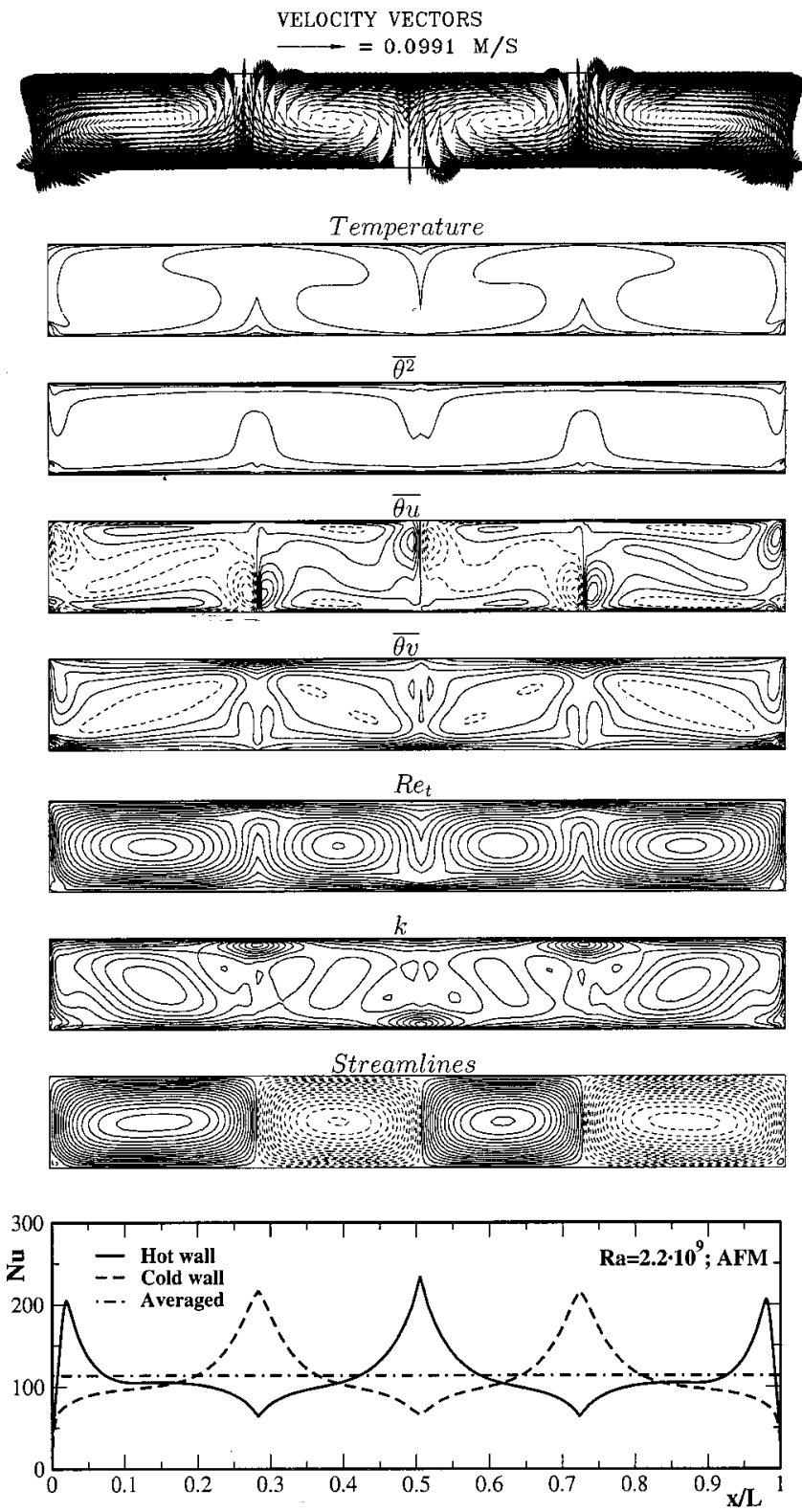

FIG. 3. AFM solutions for 1:8 enclosure heated from bottom for $\mathrm{Ra}=2.2 \times 10^{9}$.

plume jets flowing downwards and upwards between the thermally active walls.

\section{B. Integral Nusselt numbers}

The first step in the validation of the proposed turbulent heat flux model is to make a comparison of the averaged value of the $\mathrm{Nu}$ number with several experimental, DNS and LES studies for different values of the Ra number. The results are divided in two groups: simulations obtained in a range of low and moderate Ra numbers, Fig. 5, and in a range of high Ra numbers, Fig. 6. The SGDH results, compared with experimental correlations and DNS results, show a serious underprediction of $\mathrm{Nu}$ numbers. For example, at $\mathrm{Ra}=10^{6}$, the obtained SGDH Nu number is by $30 \%$ lower as compared to DNS results and represents essentially the laminar flow regime. The performance of the SGDH is even worse for the higher Ra number, such as, for example, in the case of $\mathrm{Ra}=10^{8}$. The simulations show a difference of about 

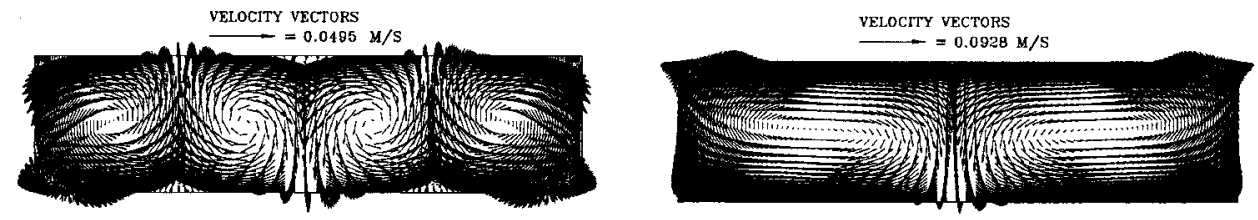

Temperature
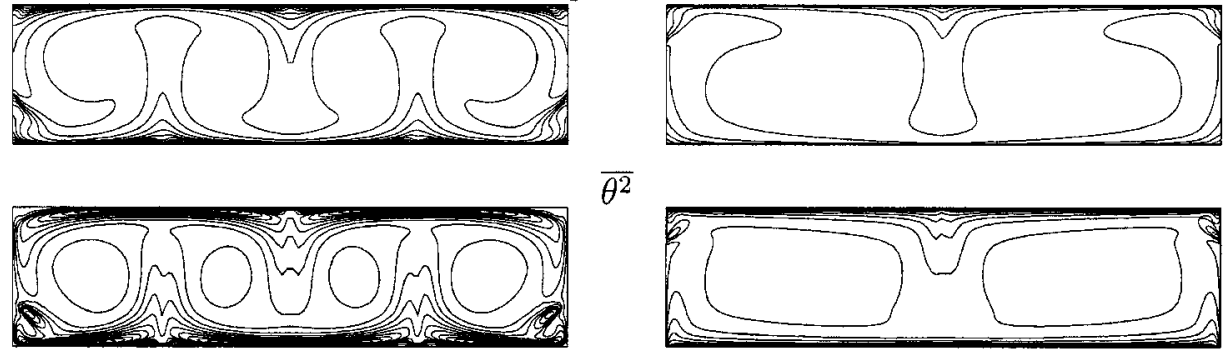

$\overline{\theta^{2}}$
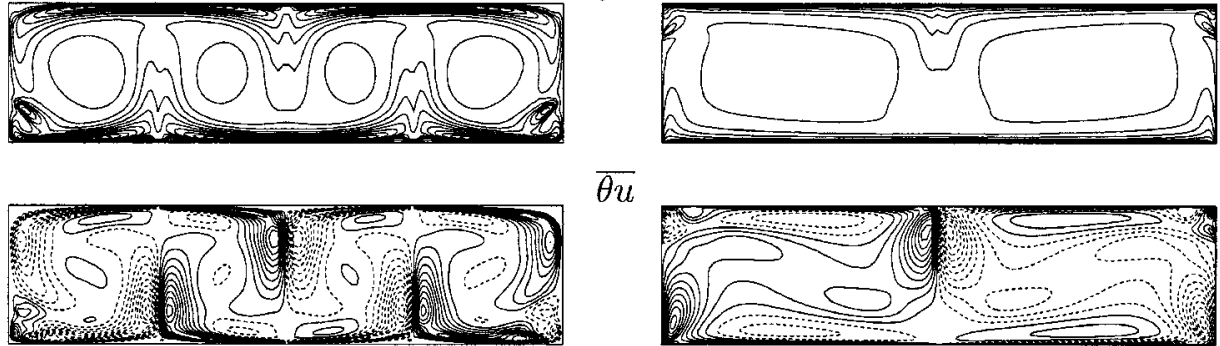

$\overline{\theta u}$
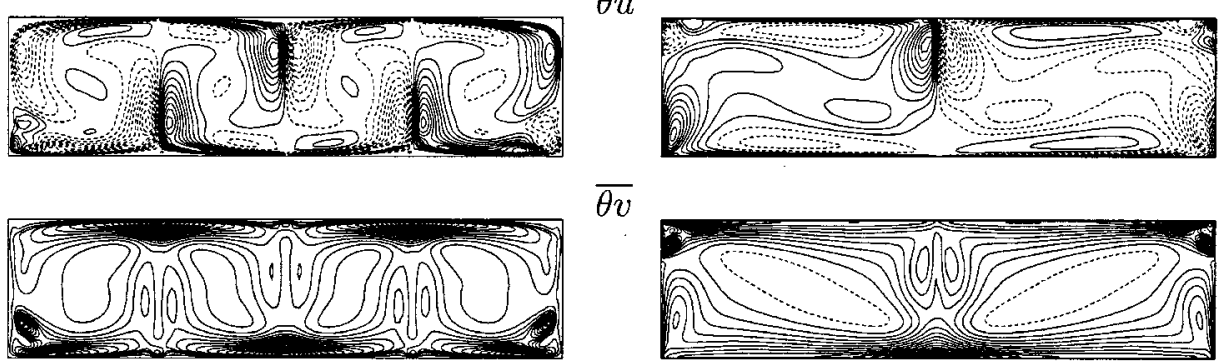

$\overline{\theta v}$
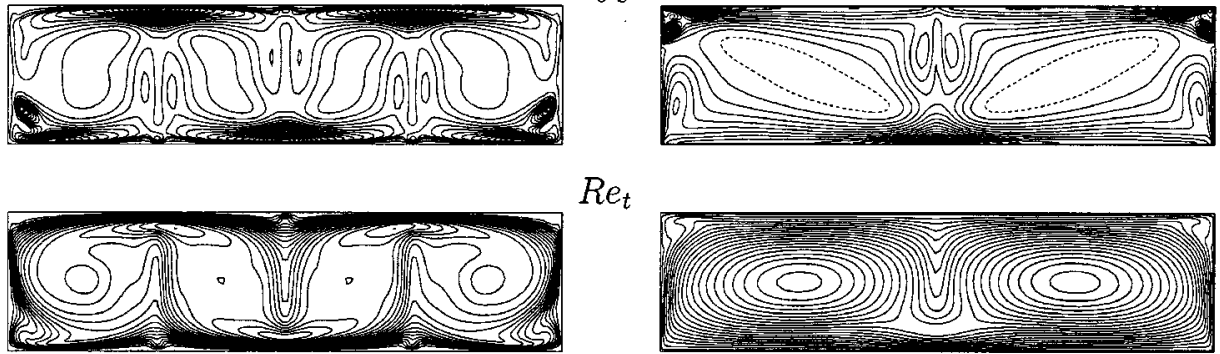

$R e_{t}$

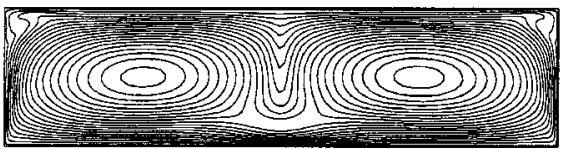

FIG. 4. AFM solutions for 1:4 enclosure heated from bottom for $\mathrm{Ra}=10^{7}$ (left) and $\mathrm{Ra}=2.2 \times 10^{9}$ (right).
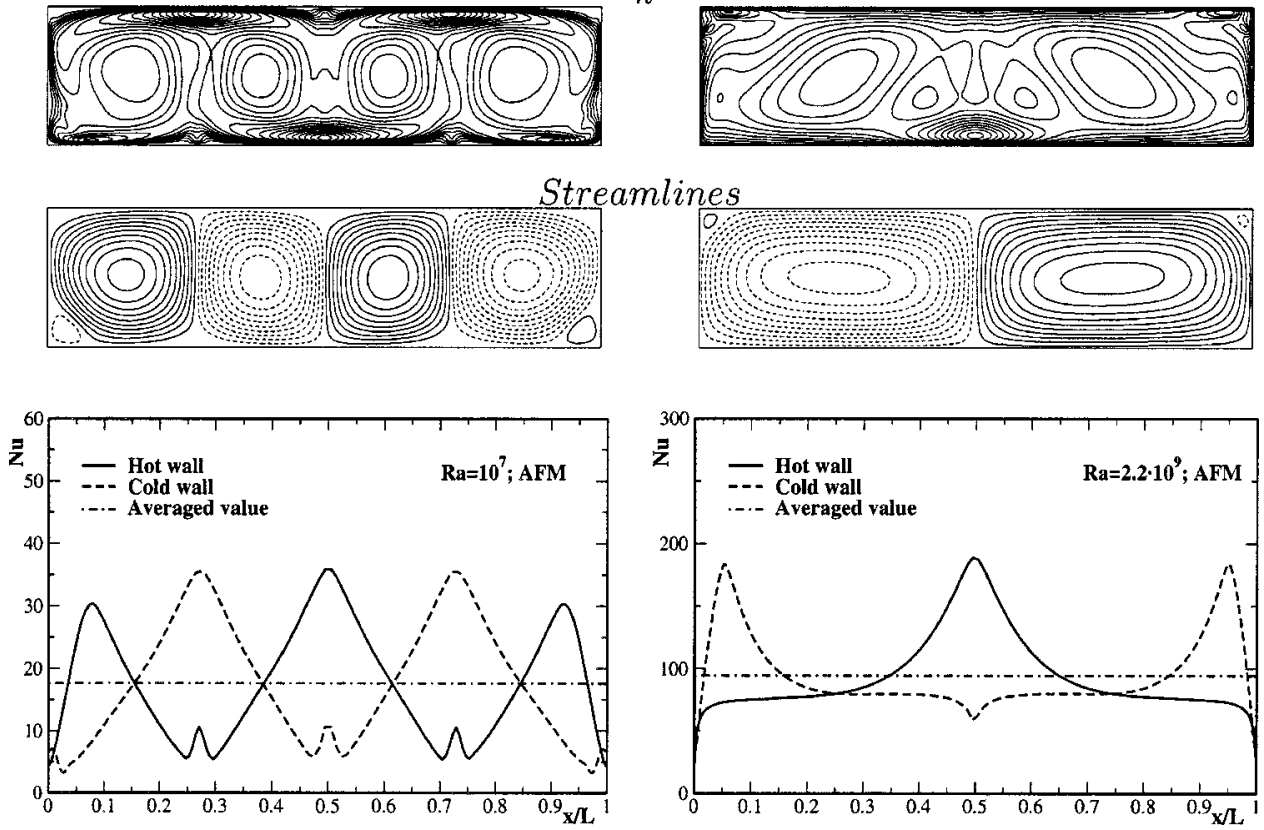

90\%. Again the simple isotropic expression failed to reproduce the turbulent heat flux and to maintain a turbulent regime. The SGDH model proved to be inadequate in the range of low and moderate Ra numbers, where even a small turbulence level can cause a drastic change in the local and, consequently, in the total heat transfer. For all cases, the AFM model was compared with several experimental correlations in the range of low Ra numbers, up to $2 \times 10^{7}$. Generally, the differences between various experimental correlations are larger than the difference between the AFM and the DNS results. In comparison with correlations of Goldstein and Chu [23], $\mathrm{Nu}=0.123 \mathrm{Ra}^{0.294}$ and $\mathrm{Wu}$ and Libchaber [24], $\mathrm{Nu}=0.146 \mathrm{Ra}^{0.286}$, the AFM computations at $\mathrm{Ra}=10^{6}$ show an overprediction of 10 and $5 \%$, respectively. It is important 


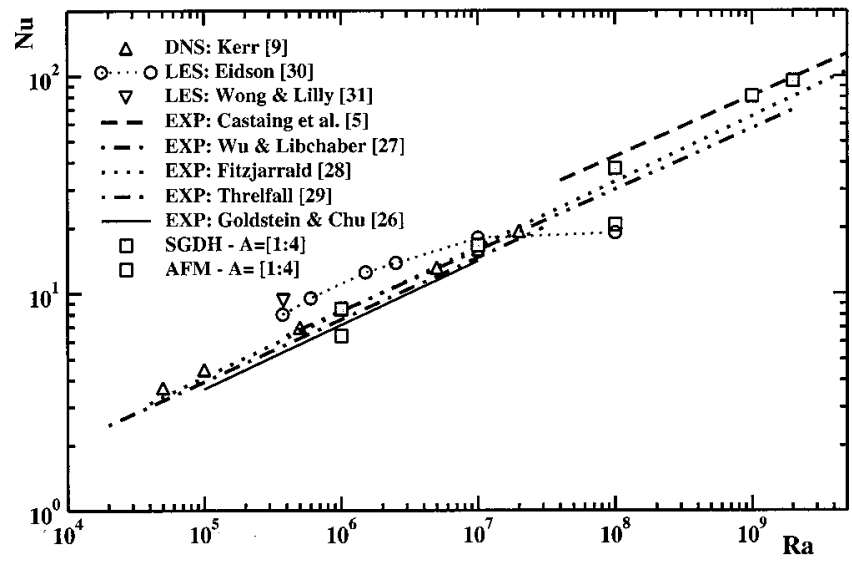

FIG. 5. Comparison of the computed $\mathrm{Nu}(\mathrm{Ra})$ results with several DNS, LES and experimental correlations for an enclosure heated from below, $H: L=1: 4, \operatorname{Pr}=0.71$, for low and moderate values of Ra numbers.

to note that those experiments were performed in enclosures of smaller aspect ratios $A=1: 1.5$ and $A=1: 1$, and, consequently, the total heat transfer correlations showed lower $\mathrm{Nu}$ numbers. In comparison with Fitzjarrald [25], Nu $=0.145 \mathrm{Ra}^{0.29}$ as well as Threlfall [27], $\mathrm{Nu}=0.173 \mathrm{Ra}^{0.28}$, and the DNS results of Kerr [7], the AFM shows excellent agreement, about $1 \%$ difference, for the same value of Ra number. Similar quality of agreement for the same set of experimental correlations and DNS data was obtained for $\mathrm{Ra}=10^{7}$. The highest difference was about $3 \%$.

In the range of moderate Ra numbers $2 \times 10^{7} \leqslant \mathrm{Ra} \leqslant 10^{9}$, at first sight it seems that the calculated values of the $\mathrm{Nu}$ number begin to deviate more from the experimental correlations of Threlfall [26] and Fitzjarrald [26], Fig. 5. The AFM overpredicts the $\mathrm{Nu}$ number by $10 \%$ at $\mathrm{Ra}=10^{8}$ in comparison with Fitzjarrald [26] correlation. On other hand, the AFM simulation results converge towards the experimental correlations of Castaing et al. [3], $\mathrm{Nu}=1+0.23 \mathrm{Ra}^{0.282}$, in the so-called "hard turbulence regime." Unfortunately, the maximal Ra number for which the DNS data are available, Kerr [7], is $\mathrm{Ra}=2 \times 10^{7}$ and, according to Castaing et al. [3], the "hard turbulence regime" starts at $\mathrm{Ra}=4 \times 10^{7}$. Much

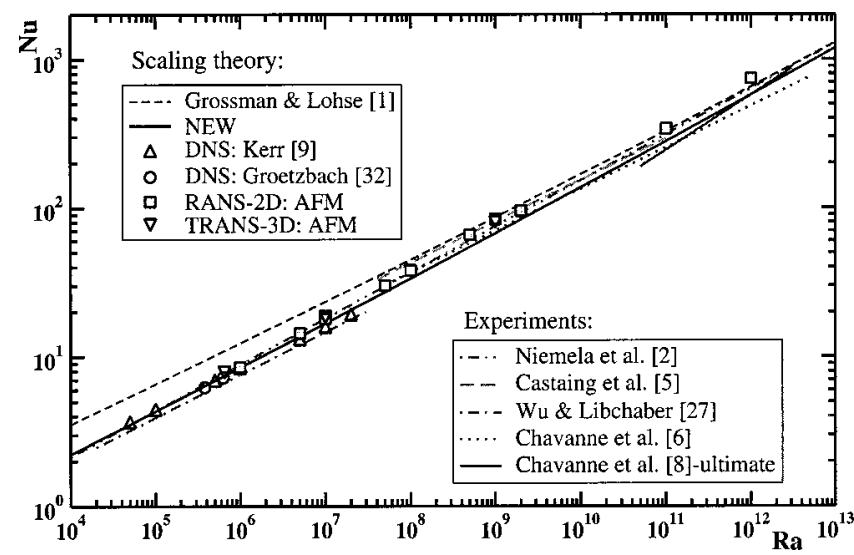

FIG. 6. Comparison of the computed $\mathrm{Nu}(\mathrm{Ra})$ results with several experimental, theoretical and DNS correlations for an enclosure heated from below, $H: L=1: 4, \operatorname{Pr}=0.71$, over a range of $\mathrm{Ra}$ numbers. desired DNS for the hard turbulence regime remains to be performed. The differences between the AFM and the Castaing et al. [3] correlation were $-7,-1$, and $2 \%$ for Ra numbers $10^{8}, 5 \times 10^{8}$, and $10^{9}$, respectively. In order to compare the results in the range of low and moderate Ra numbers that have been obtained by the AFM with other numerical techniques, the DNS results of Kerr [7] and two different sets of the LES results, Eidson [27], Wong and Lilly [28], are plotted in addition to the abovementioned experimental correlations, Fig. 5. The Eidson [27] LES results show that the LES seriously overpredicts the DNS as well as the experimental correlation by $30-40 \%$ for the Ra numbers up to $2.5 \times 10^{6}$. In addition to the Eidson [27] LES in which the Smagorinsky subgrid model is applied, more recent LES simulations of Wong and Lilly [28] were equipped with a dynamical subgrid model. Unfortunately, Wong and Lilly [28] have presented simulations for only one value of $\mathrm{Ra}=4 \times 10^{5}$ and the qualitative comparison of the predicted $\mathrm{Nu}$ number in a wider range of Ra numbers can not be performed. In comparison with Eidson's result, the results of Wong and Lilly [28] show very similar quality, with small differences between two dynamical subgrid approaches. As shown, in comparison with the Kerr [7] DNS data, as well as with experimental correlations of Fitzjarrald [25], Threlfall [26] and $\mathrm{Wu}$ and Libchaber [24], the Wong and Lilly [28] LES results seriously overpredict $\mathrm{Nu}$ numbers.

The second group of results corresponds to large values of Ra numbers, Fig. 6. To our knowledge, there are no numerical studies reported in the literature which cover such high values of Ra numbers. The performed AFM calculations show very good agreement with the Castaing et al. [3] experimental correlation, $\mathrm{Nu}=1+0.23 \mathrm{Ra}^{0.282}$. For $\mathrm{Ra}=10^{9}$ and $\mathrm{Ra}=2 \times 10^{9}$ the obtained $\mathrm{Nu}$ values of 80.6 and 95 show a difference of $1 \%$ and $2 \%$ respectively. With increasing Ra number, the AFM results start to deviate more from experimental correlations. It is important to note that with the Ra number increasing, the grid was more clustered in the vertical direction in order to resolve properly the near-wall region, so that for the highest simulated $\mathrm{Ra}=10^{12}$, the 260 grid points in the vertical direction were used. This means that any deviation from the experimental correlation cannot be regarded as a simple consequence of an insufficient grid resolution.

More recently, the Grenoble group, Chavanne et al. [4-6] repeated some of the experiments performed by the Chicago group, Castaing et al. [3], Wu and Libchaber [24], and increased Ra up to $5 \times 10^{12}$ in their experiment. These authors observed a new "ultimate" regime characterized by an increase in the heat transfer, and consequently, with an increase in the slope of the $\mathrm{Nu}(\mathrm{Ra})$ curve in the range of very large Ra numbers. For $10^{7} \leqslant \mathrm{Ra} \leqslant 3 \times 10^{10}$ the identical exponent of 2/7 was observed as in the Chicago experiment, but above the upper limit of this range the they found a continuous rise in the exponent at least up to 0.4 at the highest $\mathrm{Ra}$ $=5 \times 10^{12}$. The very recent experiments reported by Niemela et al. [29] covered even higher Ra numbers, up to $10^{17}$, but these authors did not confirm the increase in the Ra number exponent reported by the Grenoble group. Instead, they found that their measurements are correlated remarkably well by a single power law $\mathrm{Nu}=0.124 \mathrm{Ra}^{0.309}$ over eleven decades of $\mathrm{Ra}$ number, from $10^{6}$ to $10^{17}$. Our two- 
dimensional simulations with AFM show very good agreement with most recent experiments, especially with the new experimental correlation of Niemela et al. [29], Fig. 6, with a maximum discrepancies of about $10 \%$ at the highest $\mathrm{Ra}$ number of $10^{12}$.

Finally we considered the recent proposal by Grossmann and Lohse [30], who derived a unifying scaling theory that combines the $1 / 4$ and $1 / 3$ power laws for low and very high Ra numbers, respectively. Based on selection of experimental data, Grossmann and Lohse proposed the correlation $\mathrm{Nu}$ $=0.27 \mathrm{Ra}^{1 / 4}+0.038 \mathrm{Ra}^{1 / 3}$. This correlation greatly overestimates the Nusselt number obtained by DNS and more recent experiments in the low Ra number range. Adopting the same exponents for the low and high Ra number asymptotes, we recalibrated the prefactors using the best fit through the available DNS results and experiments of $\mathrm{Wu}$ and Libchaber [24] for lower Ra numbers $\left(<2 \times 10^{7}\right)$, and experiments of Castaing et al. [3], Chavanne et al. [4-6] and Niemela et al. [29] for the high and very high Ra numbers. The resulting correlation $\mathrm{Nu}=0.1 \mathrm{Ra}^{1 / 4}+0.055 \mathrm{Ra}^{1 / 3}$, shown in Fig. 5 (thick line), fits better the selected recent DNS, TRANS, and experimental results than the original expression of Grossmann and Lohse [30]. It also agrees well with the new correlation of Niemela et al. [29], $\mathrm{Nu}=0.124 \mathrm{Ra}^{0.309}$. Maximum discrepancy between these two correlations is about $8 \%$ at medium Ra numbers.

\section{Local Nusselt numbers}

We consider now in more detail the specific distribution of the local $\mathrm{Nu}$ number along horizontal walls for different values of the Ra number and aspect ratios. At the lower Ra number, $\mathrm{Ra}=10^{7}$, the Nusselt number exhibits the secondary inverse peaks for all aspect ratios considered (1:4, 1:8 and 1:16), Figs. 2 and 4, which are absent at higher Ra number. In both cases the locations of these "secondary peaks" correspond to those of plume initiation. However, some qualitative difference in the variation of the local Nusselt numbers is observed for different Ra numbers. As mentioned earlier, the wave length of the rolls increases with the Ra number. Our calculations at $\mathrm{Ra}=10^{7}$ and $10^{9}$ show an increase in wave length by factor of two indicating that this switch from one to another fundamental mode occurred in between, most probably at a distinct Ra number, which remains still to be determined.

In order to explain the possible origin of secondary inverse peaks, we plot the superimposed temperature and stream lines, as well as the distribution of velocity components and profiles at characteristic locations (at the roll edge and roll center), Fig. 7. The main difference between the solutions for the two different Ra numbers is in the distribution of the vertical and horizontal velocities at locations where the plumes are released: for the lower Ra case, Ra $=10^{7}$, both components change their sign in the region very close to the wall, indicating the existence of a small separation bubble. At the higher Ra number, $\mathrm{Ra}=2 \times 10^{9}$, this is not the case. This small recirculation at the lower Ra number at the site of plume release entrains some surrounding fluid of lower temperature and promotes locally some extra turbulence production, which, in turn, enhances local heat flux, as illustrated by higher local concentration of $k, R e_{t}, \bar{\theta}^{2}$ and
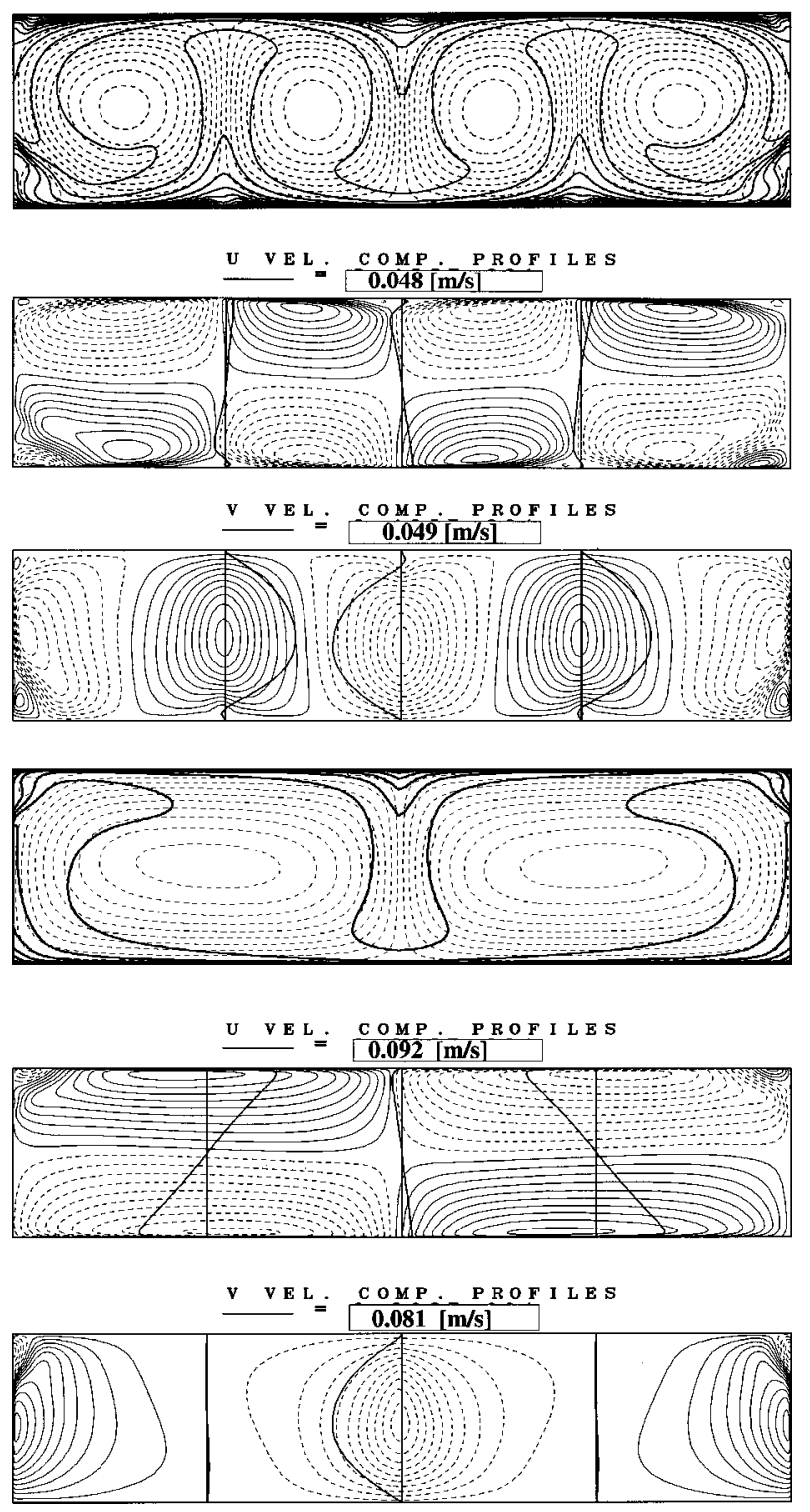

FIG. 7. The superimposed stream lines and temperature contours and the velocity components contours and profiles at characteristic locations for a 1:4 aspect ratio cavity of air for two values of Rayleigh number, $\mathrm{Ra}=10^{7}$ and $\mathrm{Ra}=2 \times 10^{9}$.

both heat flux components, $\overline{\theta u}$ and $\overline{\theta v}$. Contrary to this scenario, at the higher $\mathrm{Ra}$ the rolls have much higher velocities, which do not allow the formation of the recirculation bubble. The mixing is more intensive and all turbulence and mean properties show higher values and much milder gradients, except in the very thin conductive layer close to the walls. As a consequence, the phenomenon of the "reverse secondary peaks" is not observed. Both velocity components have a single sign across the entire height of the enclosure.

\section{Mean temperature profiles}

Figure 8 shows the distribution of the long-term averaged mean temperature (cell averaged) for a range of Ra numbers. It can be concluded that very good agreement between the mean temperature computed by AFM and DNS profiles was obtained for all Ra values calculated. This is not surprising because the calculations already showed good agreement 


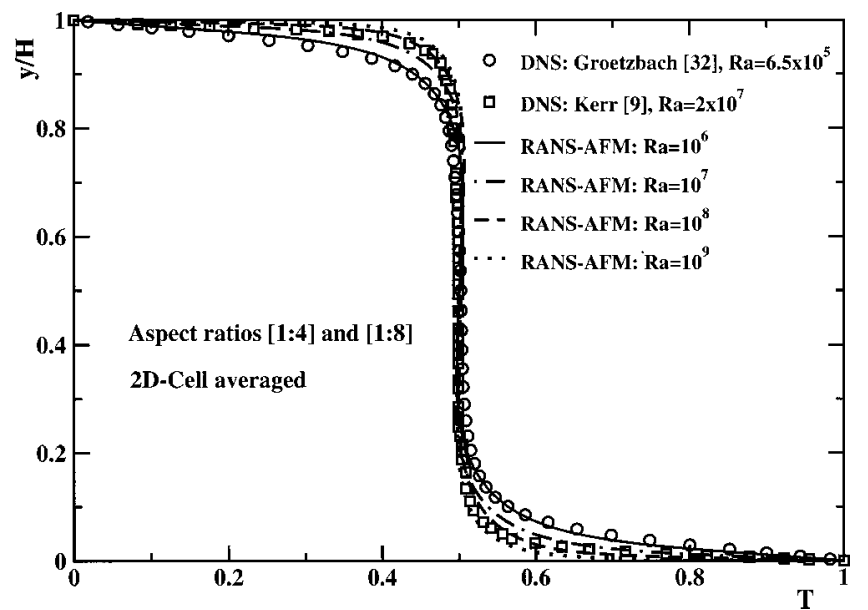

FIG. 8. Comparison of calculated mean temperature profiles with several DNS for different values of Ra numbers and different aspect ratios.

with the experimental and DNS integral Nu numbers, which are actually expressed via the temperature gradient in the near-wall region. The mean temperature profile exhibits the characteristic behavior with very steep gradients in the conductive near-wall region, and an almost isothermal core that occupies most of the space. As the Ra value increases, the boundary layers along the horizontal thermally active walls become thinner. This behavior of the mean temperature profile explains why a very fine grid resolution is needed in order to perform DNS simulation at very high Ra numbers [31]. The vertical temperature gradient is equal to zero in the central part of the cavity and, consequently, the vertical SGDH component of the turbulent heat flux is equal to zero too. This illustrates the obvious deficiency of the simple isotropic eddy diffusivity model for thermal convection heated from below.

Figure 9 shows a blowup of the near-wall temperature distributions. The agreement between the computed AFM and DNS profiles for the corresponding (or similar) Ra numbers is very good, and some small differences are the consequence of slightly differently simulated AFM and DNS Ra numbers. The overall effect of Ra number is also well repro-

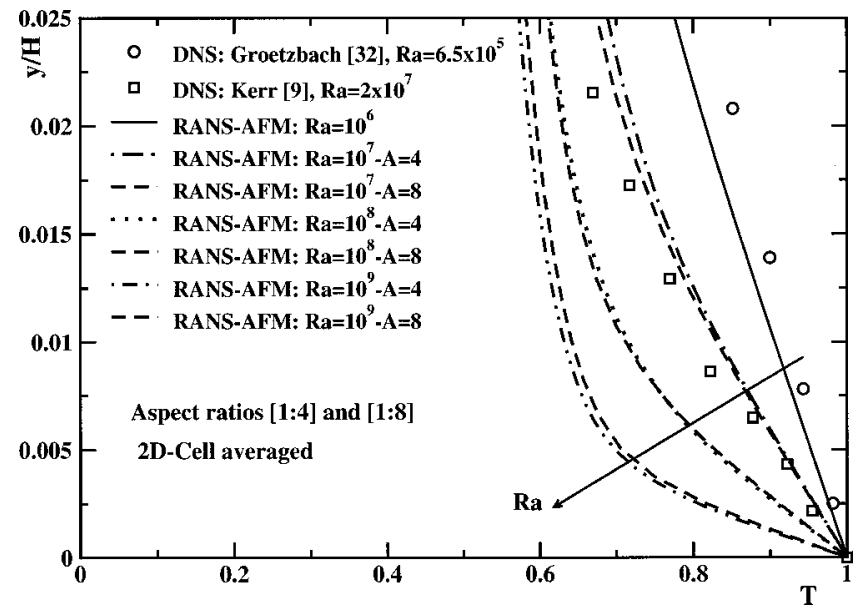

FIG. 9. The near-wall distributions of mean temperature profiles; influence of Ra number and aspect ratios. duced. The influence of different aspect ratios for the same values of Ra numbers is presented too. As seen, the different aspect ratios change the mean temperature profiles in the near-wall region only slightly. As the Ra number increases, the regions where the mean temperature gradients are significant, reduce to a very small near-wall region that occupies only up to $2 \%$ of the total cavity height (for $\mathrm{Ra}=10^{9}$ ). But this small thermal boundary layer plays a crucial role in the heat transport mechanism and must be well resolved by a numerical mesh in order to obtain correct integral values of the heat transfer coefficient.

\section{E. Effects of aspect ratio and side boundary conditions}

The wavelength of the local $\mathrm{Nu}$ number distribution reflecting the flow pattern organization increases with increasing Ra number. For the lower $\mathrm{Ra}\left(\mathrm{Ra}=10^{7}\right)$, the number of created rolls was 8 and in the case of the high Ra number, $\mathrm{Ra}=2 \times 10^{9}$ it was 4 . The obvious conclusion is that the rolls become more elongated in the horizontal direction as the $\mathrm{Ra}$ number increases. A similar conclusion was drawn by Ishiwatari et al. [8], Trompert and Hansen [14], Hewitt et al. [13], Chapman and Proctor [12].

In order to test the side wall effects, different aspect ratios were computed under the same conditions, i.e. the same boundary and initial conditions and the same level of turbulence model. The aspect ratios considered were varied in the range of 1:4 up to 1:32. In Fig. 10, the local Nu number is shown for some of the computed aspect ratios at $\mathrm{Ra}=10^{7}$ : all cases yielded identical flow patterns with a characteristic roll organization and with the size of the roll in the horizontal direction, which is equal to the vertical distance between horizontal walls. Naturally, the local $\mathrm{Nu}$ number along the horizontal walls follows the identical wavelengths. The same roll organization and the identical wavelengths are observed also in the case where side wall boundary conditions were replaced by symmetry boundary conditions, Fig. 10. It is important to note that significant computational resources were engaged in the abovementioned series of computations, especially for the higher aspect ratios, i.e. 1:16 and 1:32. The number of grid points in the vertical direction for $\mathrm{Ra}=10^{7}$ was 82. Some of the computations were repeated with an even finer distribution of 120 grid points in the vertical direction but without any significant influence on the final results. The number of grid points in the horizontal direction varied from 220 for aspect ratio 1:4 up to 820 for aspect ratio $1: 32$. The final values of the averaged $\mathrm{Nu}$ were almost identical. The difference between the results for the aspect ratios $1: 8$ and $1: 32$ were less than $0.25 \%$.

\section{F. Convective rolls creation, corner structures}

We discuss now the nature and origin of small rolls located in the corner regions, following some arguments outlined by the Princeton group, Goldhirsch et al. [15]. The main difference between these two studies is in the range of Ra numbers and applied simulation methods. The Princeton group performed two-dimensional direct numerical simulations covering Rayleigh numbers up to $\mathrm{Ra}=10^{6}$, whereas the present study uses a turbulence model, which enables to covers values up to $R a=10^{11}$. Very similar structure evolution and final flow patterns were obtained for comparable Ray- 

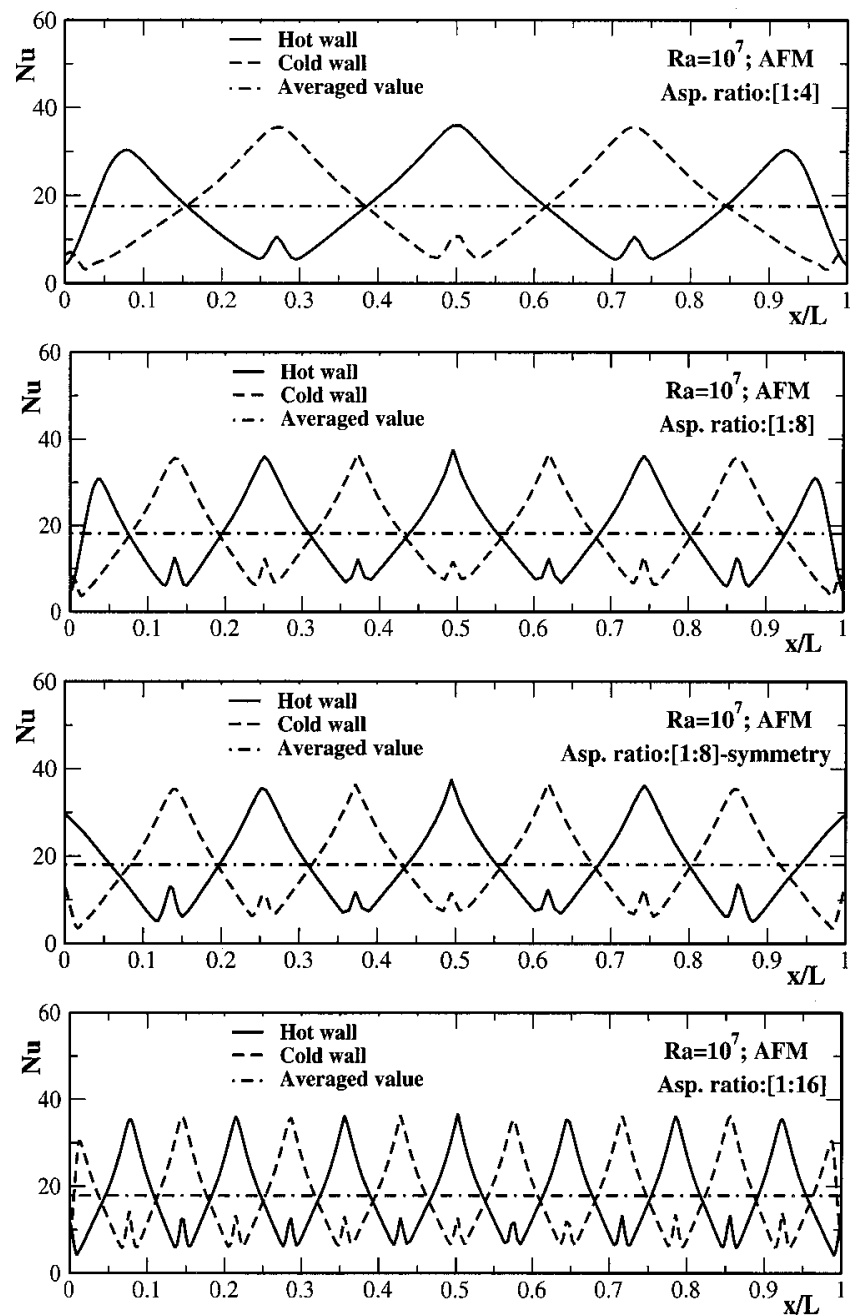

FIG. 10. Distributions of the local Nusselt numbers along the horizontal walls for AFM solutions of an enclosure heated from bottom at $\mathrm{Ra}=10^{7}$ for different aspect ratios and different side walls conditions.

leigh numbers. In order to investigate the process of the roll formation and to analyze the influence of roll structure on mechanical and thermal turbulence parameters, the closed system of equations was calculated in a transient mode for the $A=1: 4$ two-dimensional enclosure. The computations were started from uniform initial fields: all velocity components were assumed zero, turbulent quantities had very low uniform values, and the mean temperature was uniform and equal to the reference temperature $T_{r}=0.5\left(T_{h}+T_{c}\right)$, with uniform nondimensional temperature of the bottom hot wall $T_{h}=1$ and top cold wall $T_{c}=0$.

From the streamline distribution at the initial stage of heating it is obvious that the process of roll creation begins in the corner regions, Fig. 11. The four small rolls created in corners, are visible already at $\tau^{*}=10\left(\tau^{*}\right.$ $=\tau \sqrt{\beta g \Delta T H} / H)$. After creation, the roll structures continuously grow in size and begin to interact with each other as well as with the initially stagnant surrounding. As the time progresses, the roll structures move towards the interior of the enclosure causing changes in the temperature gradient in the horizontal direction and, consequently, cause an increase in the horizontal turbulent heat flux, Fig. 13. At $\tau^{*}=75$, an interesting phenomenon occurs. Until this moment, the com-

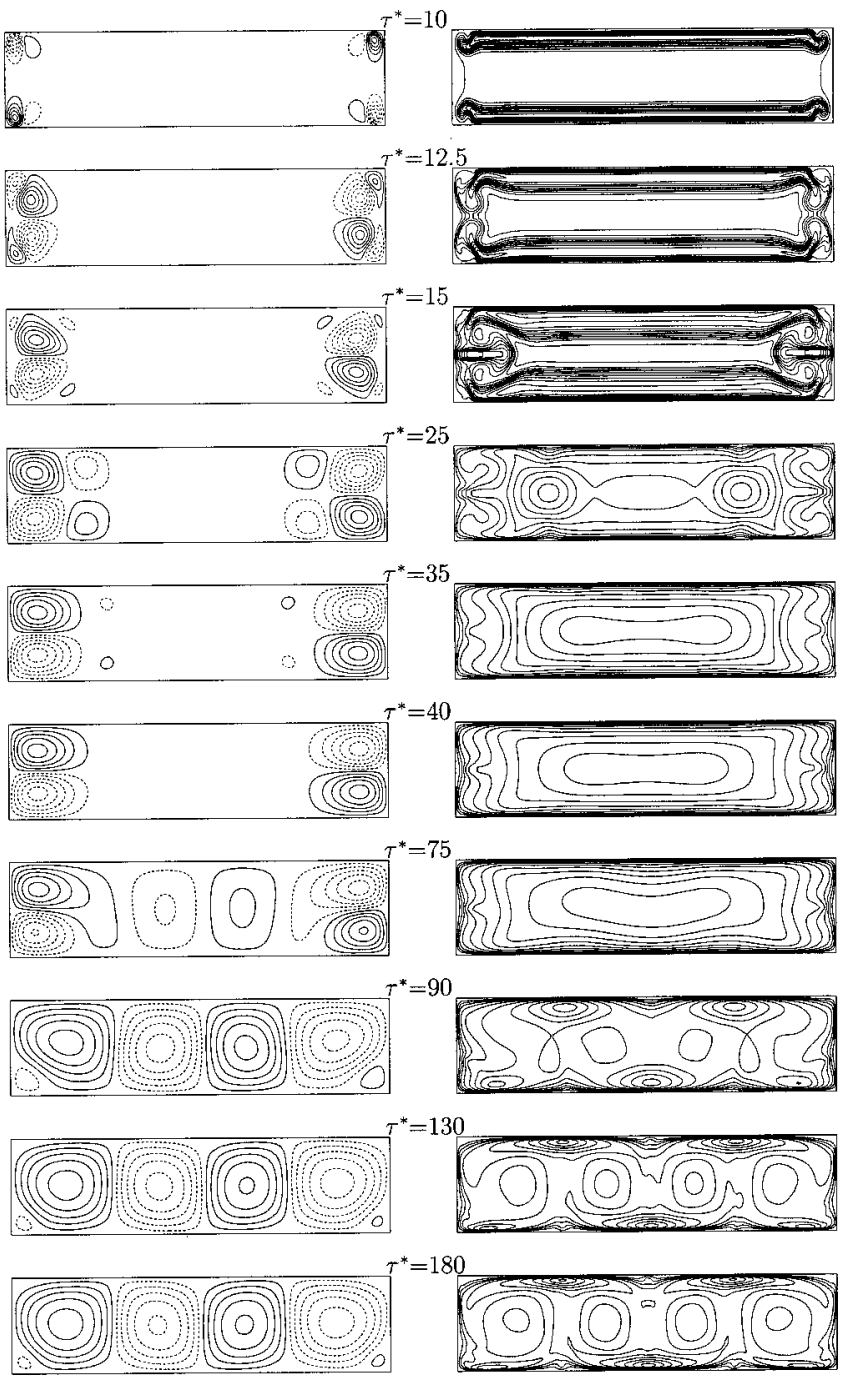

FIG. 11. Time evolution of stream lines (left) and turbulence kinetic energy (right) distributions in a $A=1: 4$ aspect ratio cavity, $\mathrm{Ra}=10^{7}, \operatorname{Pr}=0.71, \tau^{*}=\tau \sqrt{\beta g \Delta T H} / H$.

plete symmetry of the flow pattern was present with respect to the horizontal cavity centerline. Then, the rolls from the upper corner regions start to penetrate towards the bottom wall creating large rolls in the middle of the cavity. Actually, at this time the flow pattern reaches a bifurcation point. The probability is absolutely equal that the rolls from the upper regions will penetrate down, or that the rolls from the lower corners will penetrate towards the top wall. For example, the circumstance where the second situation occurs, is presented in Fig. 4. The secondary rolls become stronger and they capture primarily created corner rolls in the lower corner where they remain until the steady regime is reached. The form of the flow pattern which appears at $\tau^{*}=90$ corresponds well to the final flow pattern, but it will take additional 90 nondimensional time units, until the final steady state is reached. The dynamic behavior of the temperature fields is characterized actually by two distinctive regimes. The first one is a conductive regime present in the initial stage of the onset of the heating, and characterized by an almost linear distribution of the temperature in the vertical direction $\tau^{*} \leqslant 40$, Fig. 12. The second one is a highly turbulent convective regime characterized by the presence of roll structures in the entire 


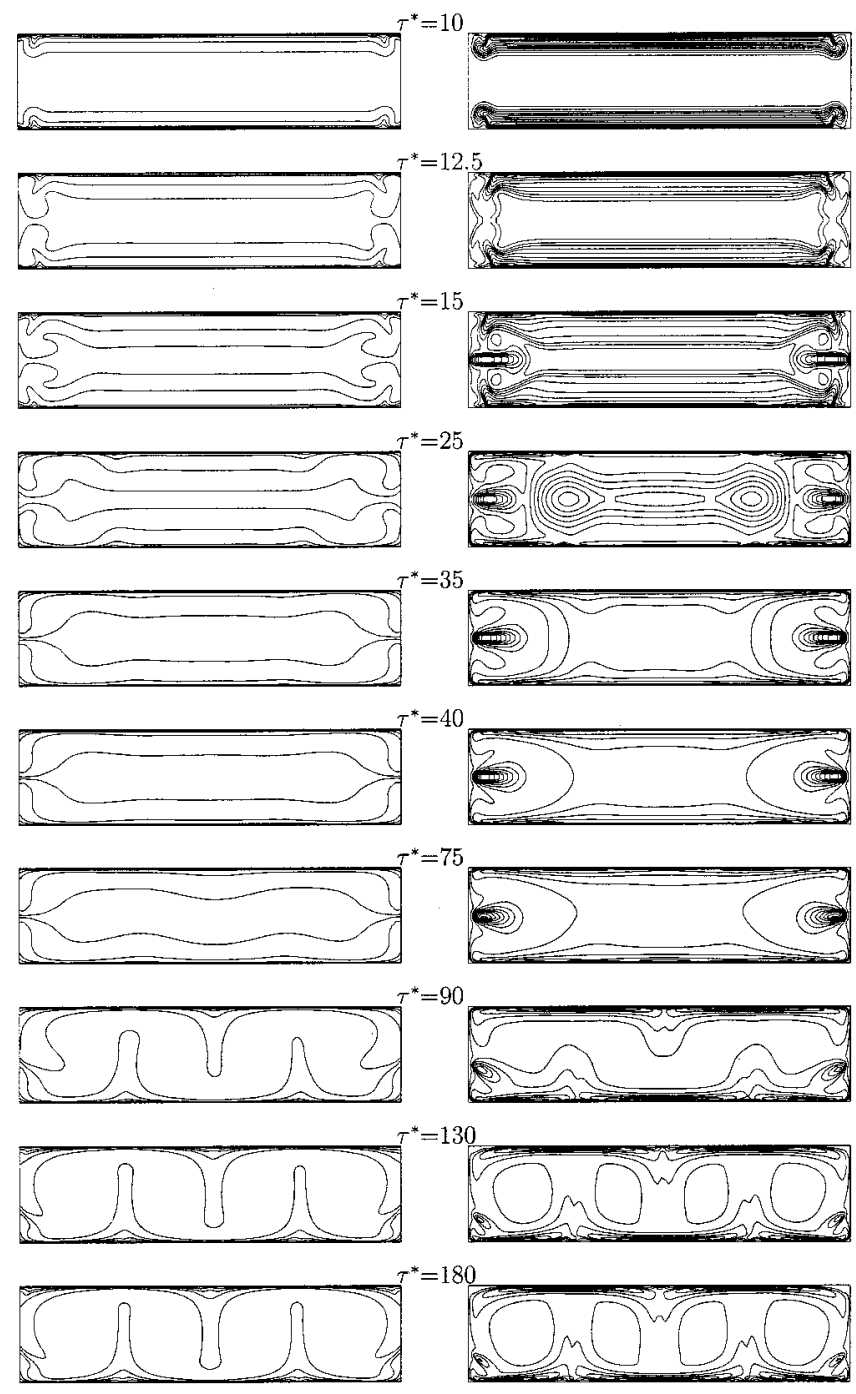

FIG. 12. Time evolution of temperature (left) and temperature variance (right) distributions in a $A=1: 4$ aspect ratio cavity, $\mathrm{Ra}$ $=10^{7}, \operatorname{Pr}=0.71, \tau^{*}=\tau \sqrt{\beta g \Delta T H} / H$.

domain of the enclosure and corresponding plume structures in between, $\tau^{*} \geqslant 75$.

In the initial stage of heating, $\tau^{*} \leqslant 15$, the turbulence kinetic energy is concentrated near the horizontal walls region, Fig. 11. As the convective roll structure grows in size and moves towards the enclosure interior, the turbulence kinetic energy spreads and eventually occupies the entire enclosure. With the formation of large secondary rolls, the centres of the maximum turbulence kinetic energy move towards the impingement regions $\tau^{*}=90$. Contrary to the situations with heating from the side, the central part of the enclosure is highly turbulent too, i.e. the turbulence is not located only in the near-wall boundary layers, but also in the enclosure core.

The vertical turbulent heat flux component and the temperature variance show a different kind of behavior, Figs. 12 and 13. They are concentrated in relatively thin boundary layers along the horizontal walls. This distribution is determined by a dominant vertical temperature gradient in the near-wall region. It is interesting to note a close dynamic coherence in behavior of the turbulence kinetic energy, temperature variance, and the vertical component of the turbulent heat flux. Finally, the steady state is reached by creating

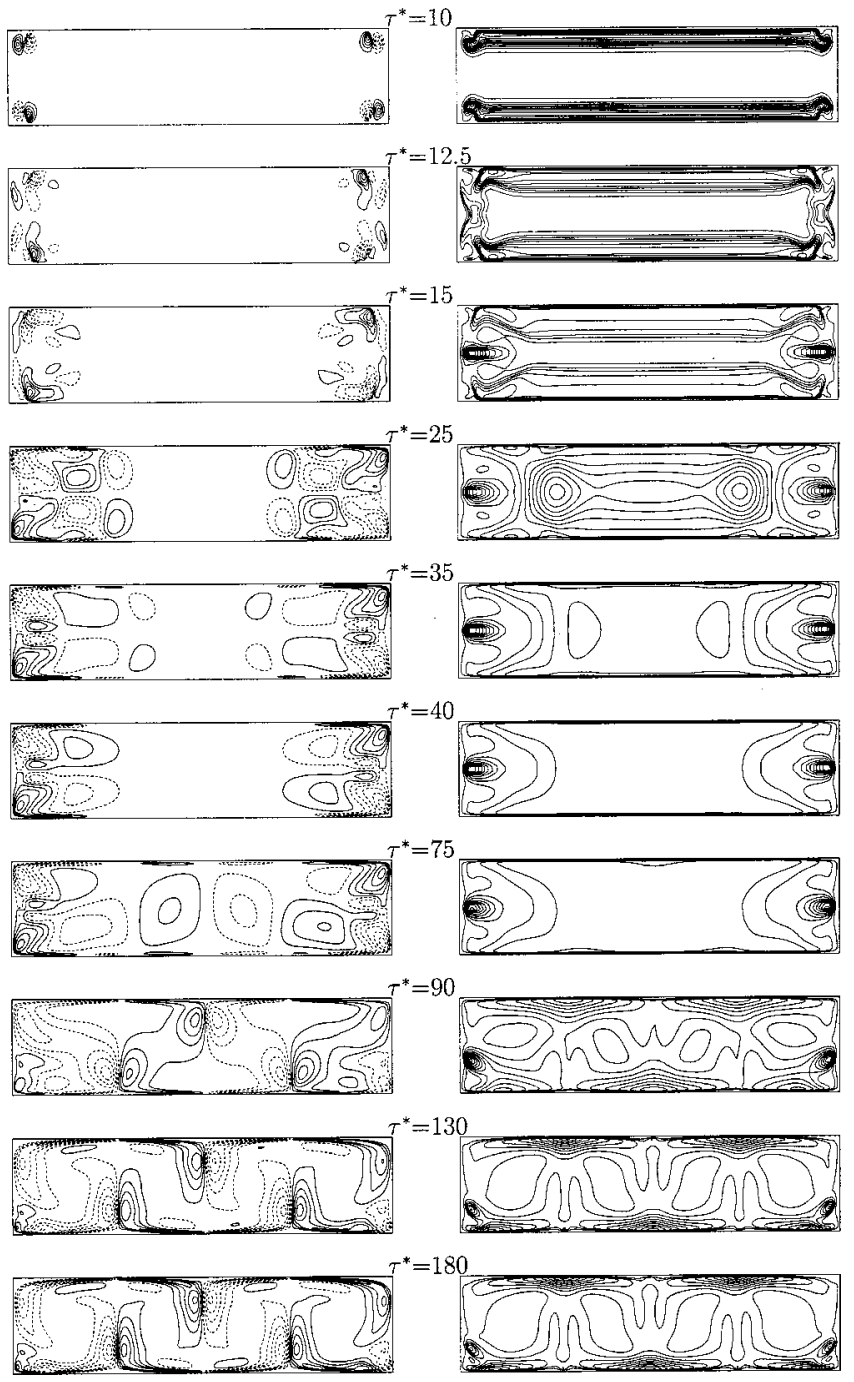

FIG. 13. Time evolution of the horizontal $\overline{\theta u}$ (left) and vertical $\overline{\theta v}$ (right) component of turbulent heat flux distributions in a $A$ $=1: 4$ aspect ratio cavity, $\operatorname{Ra}=10^{7}, \operatorname{Pr}=0.71, \tau^{*}=\tau \sqrt{\beta g \Delta T H} / H$.

a stable pattern consisting of four large rolls and two small rolls in the lower enclosure corners.

\section{CONCLUSIONS}

The turbulent thermal convection, confined in twodimensional horizontal slender enclosures heated from below, was simulated numerically by means of a single-point turbulence closure model using a two-dimensional approach. The computations were performed using an algebraic model for the turbulent heat flux (AFM) closed by a set of transport differential equations governing the turbulence kinetic energy, temperature variance and their dissipation rates. The importance of the presence of all production mechanism in the turbulent heat flux expression was demonstrated by comparison with the standard isotropic eddy-diffusivity model (simple gradient diffusion hypothesis), which shows serious deficiencies.

The computations for all $\mathrm{Ra}$ numbers and aspect ratios considered led to steady organized roll pattern in the final stage. These can be interpreted as the ensemble-averaged pattern of the essentially unsteady three-dimensional process. 
The model was tested against available DNS, LES, and experimental data in a range of low, moderate, and high $\mathrm{Ra}$ numbers. The computed long-term averaged mean and turbulence properties, roll structure morphology, local and integral wall heat transfer show all very good agreement. This agreement confirmed our conjuncture that the twodimensional approach can yield essential information about the long-term averaged flow properties and the local and integral Nusselt numbers at the bounding walls. The twodimensional approach reproduced also the ensembleaveraged roll structure in accord with the full threedimensional DNS and can be viewed as an ensembleaveraged approximation of the fully three-dimensional situation. However, a further simplification, i.e., the onedimensional approach, will completely fail in such situations, since all periodiclike roll motion will be smeared out, together with their contribution to the dynamics of turbulence and wall heat transfer. The equality of the obtained longterm averaged $\mathrm{Nu}$ numbers with DNS and experimental data revealed that the key to the success was the combination of a good near-wall modelling and resolving of roll/cell transport ("pseudomean motion").

One of the advantages of the approach followed is the ability to predict the flow and heat transfer and to capture the ensemble-averaged roll structure at high and very high Ra numbers, which are still inaccessible to direct simulation techniques DNS and LES. At such high Ra numbers the experiments are at present also limited to pointwise time measurements and long-term-averaged integral $\mathrm{Nu}$ numbers. Hence, the obtained set of computational results was used to complement the experimental data and to extend the basis for detailed analysis of the flow and heat transfer characteristics. Together with the available DNS data for low Ra numbers and the very recent experimental results at very high $\mathrm{Ra}$ numbers, the computational results were used to established a new Nu-Ra number correlation using the asymptotic lowand high-Ra numbers power laws proposed by Grossman and Lohse [31]. This correlation agrees well with the recently proposed correlation of Niemela et al. [30].

The numerical visualization of the ensemble-averaged roll structure and contour plots of mean and turbulence properties were used to explain the effects of the aspect ratios, types of side boundaries, and Ra numbers on the creation and evolution of the convective roll cells. Some peculiarities in the variation of the local heat transfer along the walls, such as the secondary peaks observed at plume release sites at low-Ra numbers, are correlated with the spatial distribution of turbulence properties and the the organization of the roll structures.

\section{ACKNOWLEDGMENTS}

The computations here reported were performed on the Delft University of Technology Cray J90SE computer and Cray C916 at the National Supercomputing Center, SARA, Amsterdam.
[1] T. Chu and R. J. Goldstein, J. Fluid Mech. 60, 141 (1973).

[2] F. Heslot, B. Castaing, and A. Libchaber, Phys. Rev. A 36, 5870 (1987).

[3] B. Castaing, G. Gunaratne, F. Heslot, L. Kadanoff, A. Libchaber, S. Thomae, X. Z. Wu, S. Zaleski, and G. Zanetti, J. Fluid Mech. 204, 1 (1989).

[4] X. Chavanne, F. Chillá, B. Chabaud, B. Castaing, J. Chaussy, and B. Hébral, J. Low Temp. Phys. 104, 109 (1996).

[5] X. Chavanne, B. Castaing, B. Chabaud, O. Chanal, F. Chillá, and B. Hébral (unpublished).

[6] X. Chavanne, F. Chillá, B. Castaing, B. Hébral, B. Chabaud, and J. Chaussy, Phys. Rev. Lett. 79, 3648 (1997).

[7] R. M. Kerr, J. Fluid Mech. 310, 139 (1996).

[8] M. Ishiwatari, S. I. Takehiro, and Y. Y. Hayashi, J. Fluid Mech. 281, 33 (1996).

[9] J. Rothermel and E. M. Agee, J. Atmos. Sci. 43, 1185 (1986).

[10] R. I. Sykes and D. S. Henn, J. Atmos. Sci. 45, 1961 (1988).

[11] C. J. Chapman, S. Childress, and M. R. E. Proctor, Earth Planet. Sci. Lett. 51, 362 (1980).

[12] C. J. Chapman, and M. R. E. Proctor, J. Fluid Mech. 101, 759 (1980).

[13] J. M. Hewitt, D. P. Mckenzie, and N. O. Weiss, Earth Planet. Sci. Lett. 51, 370 (1980).

[14] R. A. Trompert and U. Hansen, Phys. Fluids 10, 351 (1998).

[15] I. Goldhirsch, R. B. Pelz, and S. A. Orszag, J. Fluid Mech. 199, 1 (1989).
[16] S. Paolucci, J. Fluid Mech. 215, 229 (1990).

[17] K. Hanjalic, in Proceedings of the 10th International Heat Transfer Conference, edited by G. F. Hewitt (Ichem E/Taylor \& Francis, London, 1994), pp. SK-1 and SK-10.

[18] S. Kenjereš and K. Hanjalić, Int. J. Heat Fluid Flow 16, 428 (1995).

[19] K. Hanjalić, S. Kenjereš, and F. Durst, Int. J. Heat Mass Transf. 39, 1407 (1996).

[20] S. Kenjereš, Ph.D. thesis, Faculty of Applied Physics, Thermofluids Section, Delft University of Technology, Beeld en Grafisch Centrum, Delft, The Netherlands, 1999.

[21] S. A. Theerthan and J. H. Arakeri, J. Fluid Mech. 373, 221 (1998).

[22] S. A. Theerthan, Ph.D. thesis, Department of Mechanical Engineering, Indian Institute of Science, Bengalore, India, 1997.

[23] R. J. Goldstein and T. Y. Chu, Prog. Heat Mass Transfer 2, 55 (1969).

[24] X. Z. Wu and A. Libchaber, Phys. Rev. A 45, 842 (1992).

[25] D. E. Fitzjarrald, J. Fluid Mech. 73, 693 (1976).

[26] D. C. Threlfall, J. Fluid Mech. 67, 17 (1975).

[27] T. M. Eidson, J. Fluid Mech. 158, 245 (1985).

[28] V. C. Wong, D. K. Lilly, Phys. Fluids 6, 1016 (1994).

[29] J. J. Niemela, L. Skrbek, K. R. Sreenivasan, and R. J. Donnelly, Nature (London) 404, 837 (2000).

[30] S. Grossmann and D. Lohse, J. Fluid Mech. 407, 27 (2000).

[31] G. Grötzbach, J. Comput. Phys. 49, 241 (1983). 